\title{
Efficient IOT based Water Quality Prediction Using Cat Swarm Optimized Neural Network classification
}

\author{
Mrs.G.Mariammal ${ }^{1}$, Mrs.S.Venkata Lakshmi ${ }^{2}$, Mrs.C.Sathya ${ }^{3}$, A V Prabu ${ }^{4}$ \\ ${ }^{1}$ Assistant professor, Department of CSE,PSNA College of Engineering and Technology, Tamilnadu. \\ ${ }^{2}$ Assistant Professor,Department of CSE,K.L.N. College of Engineering, Tamilnadu. \\ ${ }^{3}$ Assistant professor,Department of CSE, PSNA College of Engineering and Technology, Tamilnadu. \\ ${ }^{4}$ Dept of ECE, Associate Professor,Koneru Lakshmaiah Education Foundation, Vaddeswaram, Guntur, Andhra Pradesh, India,
}

\begin{abstract}
Water is the most significant sources for human life, but, it is in serious threat of contamination by life itself. The protection and availability of drinking-water are major worries throughout the globe. In this work,anIOT based solution isintroduced to check and predict the water quality and alert the user before the water gets polluted. The proposed system uses IoT and optimized neural network for prediction. It consists of various embedded sensors like conductivity, $\mathrm{pH}$, turbidity and color. The measured sensor values are stored in the database and further directed for prediction analysis. The Cat swarm optimization (CSO) based neural network algorithm is used for forecasting thequality result. The proposed system alerts the user when any of themeasured parameters are lesser than the fixed thresholds. This technique can also be implemented in water plants, rivers and industries.
\end{abstract}

\section{Article Received: 20 September 2020, Revised: 30 November 2020, Accepted: 18 December 2020}

\section{Introduction}

Currently, water quality has become more severe threats. The quality expectation of water, as a basic part of the water environment controlling, is to find the consistency for the determined file with the time utilizing certain guaging approaches, and to understanding the development style of the water quality dependent on the past information. Presently, the estimating procedures normally utilized in water quality contains dim framework, exponential smoothing strategy, the neural organization, the numerous straight relapse, the numerical expectation model of the water quality and so forth.

The explanation is that there is an assortment of dubious impact factors inside the water condition structure. The Back Propagation Neural Network has been regularly applied as a pragmatic nonlinear incredible framework anticipating and showing mechanical get together, regardless, there are also a couple of distortions. For example, the organization structure is hard to pick, and it is essential for the organization sort out how to trap into a neighborhood least. Moreover, the learning step should be gone after for set and alter, and it is skewed to happen for a moderate social occasion or a non-mix.

In this work, an IOT based system is proposed to check and predict the water quality and alert the user earlierto avoid water contamination. The proposed system uses IoT and optimized neural network for prediction. It consists of various embedded sensors like conductivity, $\mathrm{pH}$, turbidity and color. The measured sensor values are stored in the database and further directed for prediction analysis. The cat swarm optimized neural network algorithm is used for forecasting the quality result

1. Preliminaries
IoT

Nowadays, IoT technology influencing our day to day happenings in a number of ways. IoT has got about the continuous connection between objects, people, sensors and services. The technology has attained an extensive application in all fields

Neural network

Neural networksare generally founded on the development of neurons. They include various layers with interrelated hubs. It comprises of an information layer, concealed layers and yield layer. The info layer receipts in the anticipating boundaries and the yield layer show the expectation dependent on the information. They repeat over each preparation information point and disentangle the model by giving and refreshing the weight on every hub of each layer. The prepared neural model actuates the units dependent on loads. Multi-layer perceptron (MLP) is a standard model of the neural net, which is as often as possible utilized for characterization.

$\mathrm{CSO}$

Cat swarm optimization (CSO) is a multitude Intelligence count, which was at first proposed by Chu et al. in 2006. It is roused by the standard lead of felines, and it has a novel system in showing examination and misuse stages.

General Structure of the Algorithms

The figuring makes the going with steps in order to search for ideal arrangements:

(1)Denote the cutoff points for the course of action sets. 
(2)Randomly create $\mathrm{N}$ felines (plan sets) with arbitrary speed .The speed ought to be littler than a fixed most prominent speed esteem.

(3)Randomly portray the felines into looking for and following modes .From all out populace, $20 \%$ felines considered following mode and $80 \%$ felines took into consideration looking for mode. response .

(4)Evaluate the fitness function for desired

(5)The cats at that point move to either seeking or tracing mode.

(6) Check final solution obtained. If desired output reached terminate the iteration otherwise repeat step 2 to 5 .

In any case, objective of cat swarm is defined by :

$\mathrm{P}_{\mathrm{i}}=\frac{\left\|\mathrm{FS}_{\mathrm{i}}-\mathrm{FS}_{\mathrm{b}}\right\|}{\mathrm{FS}_{\text {max }}-\mathrm{FS}_{\text {min }}}$, where $0<\mathrm{i}<\mathrm{j}$

If the objective is minimization, then $\mathrm{FS}_{\mathrm{b}}=\mathrm{FS}_{\max }$; otherwise, $\mathrm{FS}_{\mathrm{b}}=\mathrm{FS}_{\text {min }}$

\section{Related work}

Li et al 2015 have studied the difficulties of water quality predictionand introduced an ANN-Markov chain methodology to forecast the quality of water. The implementation and evaluation performed on data set with 27 years of Tolo Harbor for train and testing.

Pop et al 2014 have presented a biologically inspired method to forecast water quality and studied the effect of applying a temporal series method for specific case prediction. The proposed prediction uses neural networks for decision support.Lee YootKhuan et al 2002 haveinvestigated the efficiency of ANN models for forecasting the Water Quality Index for waterways in Malaysia. The proposed neural model was trained by seven main parameters for the forecasting of the Water Pollutant Index, Water Quality Index and Water Quality Class.

Yu, L et al 2009 have proposed new fractal model to predict water quality. The original data is converted into a fractal model of $\mathrm{D} \mathrm{N}(\mathrm{r})=\mathrm{C} / \mathrm{r}$, and variable dimension model is created. By utilizing variable dimension fractal, the stability of pollutant can be forecasted.Lu, J et al 2009 have introduced a decision-making tree-basedchlorophyll level prediction in water toallow online observing of data, combined with the algal growth dynamics,mean and average standard deviation has been calculated.Ruijian Zhang et al 2011have developed an accurate and low-cost approach for water quality prediction. The implementation results exhibitedthat using data mining models for water quality prediction is a very useful directionfor Indiana's water quality assessment.

Joslyn, K et al 2018 haveused two supervised regression algorithms of support vector regression and gradient for water quality prediction. They demonstrated the effect of trainedregressor to detect sensor faults in the form of a linear scaling

Wang, X et al 2010 haveproposed water quality and bloom prediction system using neural network. It can realize the change of water quality by neural network for water bloompredictionsimultaneously, thusresolve the lagging state of the quality monitor and the difficulty of water bloom forecasting.

Yanfei Zhao et al 2015have proposed another forecast model by joining Kalman channel and Back Propagation Neural Network. The recreation result shows that the proposed technique can viably build the forecast precision and end up being superior to different strategies.

Wu, D et al 2019 have proposed a tensor model for water quality valuation. This model predicts quality by the dimensions of water quality parameter, locations and time. Additionally, the Random Forest algorithm applied to classify the quality level

Wang, Y et al 2017 have proposed another water quality forecast strategy utilizing long and momentary memory neural organization. At first, an anticipating model dependent on neural organization is set up. At that point, to expand the prescient precision of the model, a progression of reproductions and boundaries choice are performed.

Xiang, Y 2009 el al have proposed water quality prediction model through the use of LS-SVM. To overcome the drawback of the BP algorithm, least squares support vector machine (LS-SVM) integrated with particle swarm optimization (PSO).

Chi Zhou et al 2006 have introduced a Particle Swarm Optimization (PSO) based Artificial Neural Network (ANN) for quality prediction. The PSO optimization used to train the neural network for further accuracy improvement.

\section{Proposed system}

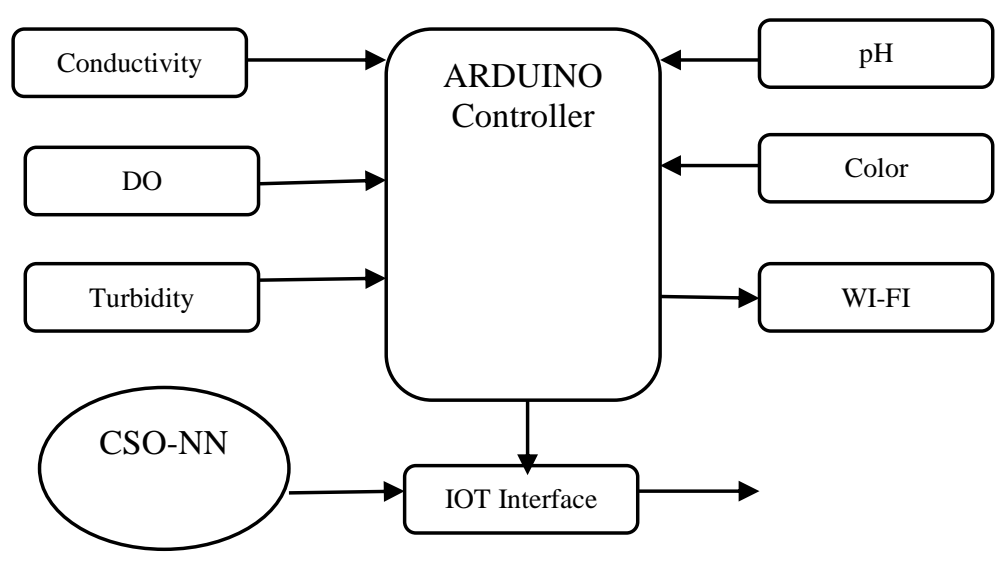

Figure 1:Proposed system 
In the proposed system integrates various embedded sensors such as $\mathrm{pH}$ sensor, coloursensor, Turbidity sensor, conductivity sensor and DO sensor etc. A Wi-Ficomponent is used to transfer data from arduino to cloud server. The sensors unceasingly transfer the data to the controller.The collected data is processed by optimized neural classifier and alert user to avoid water contamination earlier.

Controller

\section{Hardware design}

An Arduino UNO is used as a core one. The Arduino victimized here is mega 328 because of multiple analog pins.

Sensors for monitoring

$\mathrm{pH}$ sensor $-\rightarrow$ used to monitor $\mathrm{pH}$ levels it measures the hydrogen-ion density in a bleach

Temperature sensor --) LM35 for measuring temperature

Wi-Fi module $\rightarrow$ wireless meshwork that is connected to the Internet

IoT platform and Neural network models $\rightarrow$ The quality boundaries are marked datasets with wanted yields of a particular blend of information sources. The neural organization will create yield to arrange water quality as risky, be cautious, and acceptable.

Proposed CSO-NN

There are numerous methods for training a neural network. By far the most general technique is called backpropagation. Though mathematically elegant, backpropagation is not suitable. This work designates an alternative neural network training technique that uses CWO. In proposed CSO optimized NN, weights and bias of both hidden and output layer updated using CSO algorithm. And error is displayed before and after optimization by CSO.

Implementation results

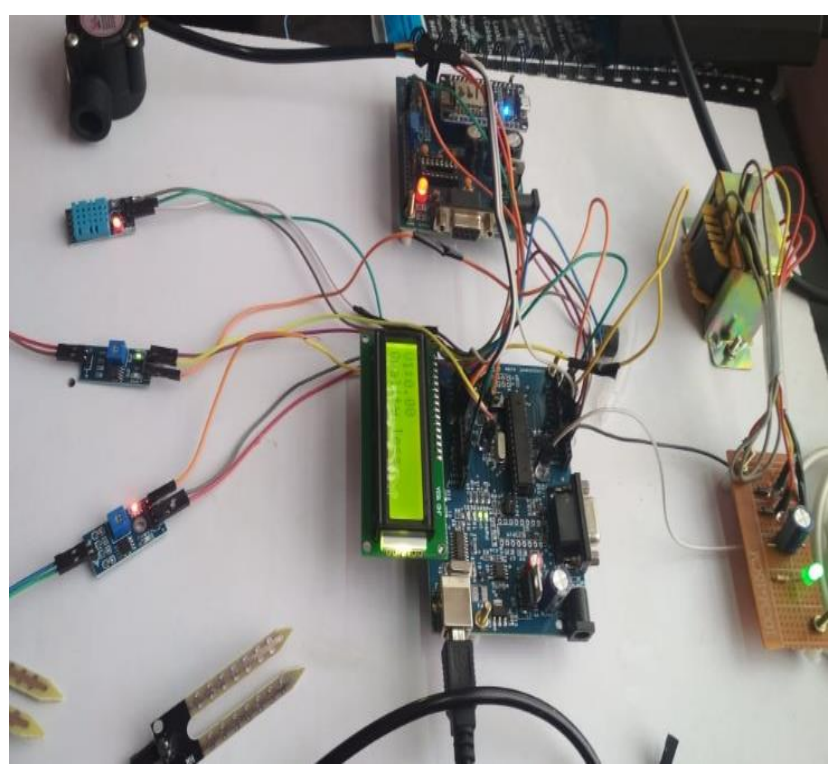

Figure 2:Harware implementati

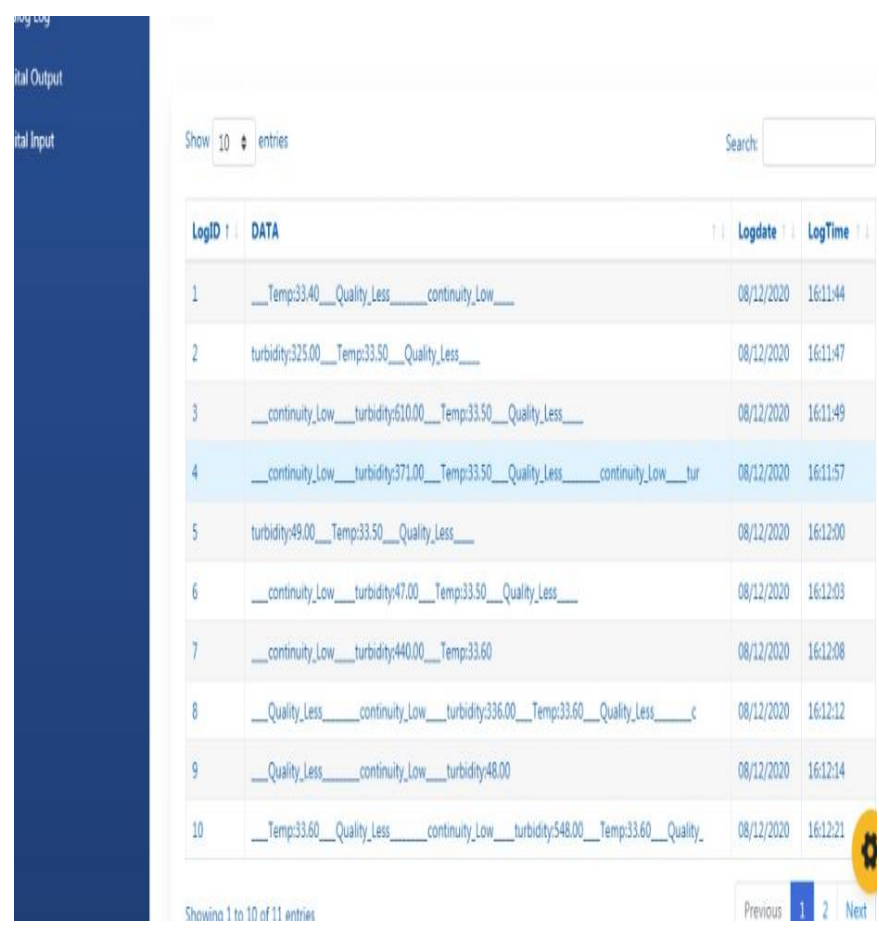

FIGURE 3 : on results and IOT server access

Figure 2 shows the overall hardware implementation of proposed IOT based water quality prediction. The measure values displayed in LCD and updated in IoT server. Table.1 shows prediction accuracy results of proposed CSO-NN with other classification algorithms

Table1: Performance comparison

\begin{tabular}{|l|l|l|}
\hline s.no & Method & $\begin{array}{l}\text { Accuracy } \\
\text { percentage }\end{array}$ \\
\hline 1 & SVM & 89.2 \\
\hline 2 & NN & 91.6 \\
\hline 3 & CSO-NN & 93.4 \\
\hline
\end{tabular}

\section{Conclusion}

The work presents the low cost and accurate water quality prediction system to avoid pollution of water. The contamination status of water is examined using sensorbased IoT system and the future forecasting of water pollution is obtained using optimized neural classification. The proposed IOT solution comprises of sensors connected to the IoT server to analyze the condition of water. And the warning data is sent to the client before the water gets over pollution. The proposed predicting and alerting system supports to save the water from pollution and is also inexpensive

\section{References}

[1] Zhou, C., Gao, L., Gao, H., \& Peng, C. (2006, June). Pattern classification and prediction of water quality by neural network with particle swarm 
optimization. In 2006 6th World congress on intelligent control and automation (Vol. 1, pp. 2864-2868). IEEE.

[2] Xiang, Y., \& Jiang, L. (2009, January). Water quality prediction using LS-SVM and particle swarm optimization. In 2009 Second International Workshop on Knowledge Discovery and Data Mining (pp. 900-904). IEEE.

[3] Wang, Y., Zhou, J., Chen, K., Wang, Y., \& Liu, L. (2017, November). Water quality prediction method based on LSTM neural network. In 2017 12th International Conference on Intelligent Systems and Knowledge Engineering (ISKE) (pp. 1-5). IEEE.

[4] Wu, D., Wang, H., \&Seidu, R. (2019, August). A Tensor Model for Quality Analysis in Industrial Drinking Water Supply System. In 2019 IEEE Intl Conf on Dependable, Autonomic and Secure Computing, Intl Conf on Pervasive Intelligence and Computing, Intl Conf on Cloud and Big Data Computing, Intl Conf on Cyber Science and Technology Congress (DASC/PiCom/CBDCom/CyberSciTech) (pp. 1090-1092). IEEE.

[5] Zhao, Y., Zou, Z., \& Wang, S. (2015, August). A Back Propagation Neural Network Model based on kalman filter for water quality prediction. In 2015 11th International Conference on Natural Computation (ICNC) (pp. 149-153). IEEE.

[6] Wang, X. Y., Xu, J. P., Liu, Z. W., Dai, J., \& Zhu, S. P. (2010, March). An Intelligent System on Water Quality Remote Monitor and Water Bloom Prediction. In 2010 International Conference on Measuring Technology and Mechatronics Automation (Vol. 1, pp. 521-524). IEEE.

[7] Joslyn, K., \&Lipor, J. (2018, December). A Supervised Learning Approach to Water Quality Parameter Prediction and Fault Detection. In 2018 IEEE International Conference on Big Data (Big Data) (pp. 2511-2514). IEEE.

[8] Zhang, R., Zhao, H., \&Piao, Y. (2011, January). Applying data mining and hpc for water quality assessment and prediction. In 2011 3rd International Conference on Advanced Computer Control (pp. 487-491). IEEE..

[9] Lu, J., \& Huang, T. (2009, August). Data mining on forecast raw water quality from online monitoring station based on decision-making tree. In 2009 Fifth International Joint Conference on INC, IMS and IDC (pp. 706-709). IEEE.

[10] R. Sathish, R. Manikandan, S. Silvia Priscila, B. V. Sara and R. Mahaveerakannan, "A Report on the Impact of Information Technology and Social Media on Covid-19," 2020 3rd International Conference on Intelligent Sustainable Systems (ICISS), Thoothukudi, India, 2020, pp. 224-230, doi: 10.1109/ICISS49785.2020.9316046.

[11] Yu, L., \& Wang, J. (2009, April). Water quality prediction based on fractal theory. In 2009
International Joint Conference on Computational Sciences and Optimization (Vol. 1, pp. 315-317). IEEE.

[12] Manikandan, R and Dr.R.Latha (2017). "A literature survey of existing map matching algorithm for navigation technology. International journal of engineering sciences \& research technology", 6(9), 326-331.Retrieved September $15,2017$.

[13] Khuan, L. Y., Hamzah, N., \&Jailani, R. (2002, July). Prediction of water quality index (WQI) based on artificial neural network (ANN). In Student Conference on Research and Development (pp. 157-161). IEEE.

[14] Pop, F., Ciolofan, S., Negru, C., Mocanu, M., \&Cristea, V. (2014, July). A bio-inspired prediction method for water quality in a cyberinfrastructure architecture. In 2014 Eighth International Conference on Complex, Intelligent and Software Intensive Systems (pp. 367-372). IEEE.

[15] Li, X., \& Song, J. (2015, July). A New ANN-Markov chain methodology for water quality prediction. In 2015 International Joint Conference on Neural Networks (IJCNN) (pp. 1-6). IEEE. 